\title{
RISCO NUTRICIONAL NO PERÍODO PRÉ-OPERATÓRIO
}

\author{
Nutritional risk in the preoperative period
}

\author{
Vânia Aparecida LEANDRO-MERHI, José Luis Braga de AQUINO, José Francisco Sales CHAGAS
}

\begin{abstract}
ABCDDV/660
Leandro-Merhi VA, Aquino JLB, Chagas JFS. Risco nutricional no período pré-operatório. ABCD Arq Bras Cir Dig 2009;22(3):143-6

RESUMO - Racional - Prognóstico nutricional ruim no período pré-operatório -, principalmente nos pacientes que apresentam perda de peso -, pode levar à complicações pós-operatórias em maior frequência. Objetivo - Investigar indicadores de risco nutricional em pacientes internados em enfermaria de cirurgia, no período pré-operatório, estratificados por grupos de doenças mais frequentes no serviço. Método - Foram avaliados 512 pacientes portadores de abdome agudo, doenças do trato digestório, doenças de vias biliares, doenças ginecológicas, doenças vasculares, hérnias e traumas, sendo analisados o índice de massa corporal, a perda de peso recente, o consumo energético habitual, a contagem de linfócitos e o tempo de internação. Os dados foram comparados entre os grupos de doenças, sendo utilizado o teste qui-quadrado para a comparação de proporções ou teste exato de Fisher, quando necessário, com nível de significância de 5\%. Resultados - Os pacientes com trauma, seguidos daqueles portadores de doenças do trato digestório e abdome agudo, apresentaram índice de massa corporal mais baixos, com diferença significativa entre os grupos $(P=0,0222)$, e entre os que mais apresentaram perda de peso recente na internação, estavam os pacientes com abdome agudo, $(P=0,0048)$. Verificouse maior percentual de depleção imunológica grave e moderada nos portadores de abdome agudo $(\mathrm{P}<0,0001)$ e, maior percentual de pacientes com consumo energético inferior a 1000 kcalorias, entre aqueles com doenças vasculares, $(P=0,0311)$. Os pacientes com trauma apresentaram maior tempo de internação $(\mathrm{P}<0,0001)$. Conclusão - Os pacientes portadores de abdome agudo e trauma apresentaram maior risco nutricional no período pré-operatório, o que deveria demandar estratégias de atenção nutricional logo no início da internação.

DESCRITORES - Estado nutricional.
\end{abstract}

\section{INTRODUÇÃO}

Vários estudos já têm documentado previamente o risco nutricional que pacientes cirúrgicos demonstram no período pós-operatório, quando apresentam prognóstico nutricional ruim no período pré-operatório, principalmente nos que têm perda de peso. A literatura tem mostrado claramente que a desnutrição é um fator de risco significativo de complicações pós-operatórias, principalmente em operações abdominais ${ }^{1,7,21,24}$. Tanto os pacientes de clínica médica, como os de clínica cirúrgica, estão expostos aos fatores de risco para o desenvolvimento de desnutrição hospitalar. Alterações do trato digestório, dificuldades para cobrir as necessidades nutricionais e outros fatores associados ao curso clínico da doença são exemplos. Do ponto de vista nutricional, os pacientes cirúrgicos podem apresentar pior prognóstico em função dos longos períodos de jejum a que são submetidos, no pré e pós-operatórios, além de outras complicações oriundas de operações gastrointestinais.

Estudos têm evidenciado dados diferentes sobre presença ou ausência de desnutrição em pacientes cirúrgicos e não cirúrgicos ${ }^{3,6,9,12}$. Recentemente Pacelli, et al. ${ }^{19}$ avaliando a incidência de mortalidade e complicações

Trabalho realizado na Faculdade de Nutrição e Medicina, Puc-Campinas,SP, Brasil

Endereço para correspondência: Vânia Ap. Leandro-Merhi, e-mail: valm@dglnet.com.br pós-operatórias e o estado nutricional com indicadores nutricionais de rotina, mostraram que a perda de peso e a hipoalbuminemia não foram associados ao aumento do risco de morbidade e mortalidade em pacientes submetidos à operações gástricas. Ao longo destas constatações, é necessário avaliar ainda se a desnutrição está ou não presente nestes pacientes, se há alguma influência no tempo de internação, ou se estes pacientes, ao contrário de desnutrição, estão apresentando algum quadro de eutrofia ou obesidade, considerando a transição nutricional que está sendo vivenciada. É importante estabelecer rotinas de monitoramento nutricional nos hospitais ${ }^{10}$, por meio de estratégias adequadas e eficientes e com a aplicação de protocolos de investigação clínica e nutricional, na tentativa de reconhecer o real estado nutricional do paciente ao internar-se.

Frente a estas constatações, o presente estudo tem como objetivo investigar indicadores de risco nutricional em pacientes internados em enfermaria cirúrgica, no período pré-operatório, estratificados por grupos de doenças mais frequentes no serviço.

\section{MÉTODO}

Este estudo foi conduzido em serviço de cirurgia geral e de trato digestório no Hospital e Maternidade Celso Pierro da Pontifícia Universidade Católica de Campinas, após obtenção de parecer favorável do Comitê de Ética em Pesquisa da Instituição (Protocolo n ${ }^{0}$ 504/05). 
Todos os pacientes internados na enfermaria de Clínica Cirúrgica, no período entre março e novembro de 2007, foram inicialmente considerados aptos a participar da pesquisa ( $\mathrm{n}=928$ pacientes). A partir disto, foram incluídos aqueles em número suficiente para análise em cada grupo de doença, permanecendo então 512 pacientes de ambos os sexos ( $50,2 \%$ do sexo feminino e $49,8 \%$ do sexo masculino), portadores das seguintes condições clínicas: abdome agudo $(\mathrm{n}=52)$; doenças do trato digestório - esôfago, estômago e intestino $(\mathrm{n}=83)$; doenças de vias biliares $(\mathrm{n}=62)$; doenças ginecológicas $(\mathrm{n}=119)$; doenças vasculares $(\mathrm{n}=91)$; hérnias $(\mathrm{n}=56)$ e traumas $(\mathrm{n}=49)$. Não houve limite máximo de idade, enquanto que para a idade mínima, foram excluídos os pacientes com idade inferior a 20 anos. $\mathrm{O}$ estudo foi do tipo transversal, com a coleta realizada no período pré-operatório e nas primeiras 24 horas de internação, mediante a aplicação de um protocolo de atendimento nutricional previamente definido, com o preenchimento das informações de identificação e diagnóstico a partir do prontuário médico. Além dos dados de identificação, o protocolo era composto por dados antropométricos, laboratoriais, dietéticos e o tempo de internação.

Para a identificação do estado nutricional na internação, foram coletados indicadores como índice de massa corporal (IMC), perda de peso recente, contagem de linfócitos e o consumo energético habitual, a partir da investigação da história alimentar e o tempo de internação. $\mathrm{O}$ índice de massa corporal foi calculado por meio do quociente peso/ estatura $^{2}$ e classificado obedecendo aos critérios estabelecidos pela $\mathrm{WHO}^{27}$ para adultos (até 60 anos) e Lipschitz ${ }^{15}$ para idosos (> 60 anos). A perda de peso recente foi classificada como sim, para aqueles pacientes que haviam apresentado perda de peso durante a internação e como não, para os que não a apresentaram.

Para a análise da contagem de linfócitos, utilizou-se como ponto de corte os valores de referência ${ }^{5,16}$, sendo os linfócitos também divididos por faixas de depleção nutricional como: sem depleção, acima de $1500 \mathrm{cel} / \mathrm{mm}^{3}$; depleção leve, de $1200-1500 \mathrm{cel} / \mathrm{mm}^{3}$; depleção moderada, de $1200-800 \mathrm{cel} / \mathrm{mm}^{3}$; depleção grave, $<800 \mathrm{cel} / \mathrm{mm}^{3}$.

O consumo energético habitual foi estratificado por faixas de consumo energético em: até 1000 kcalorias; $>1000-1500$ kcalorias; $>1500-2000$ kcalorias; > 2000-2500 kcalorias e superior a 2500 kcalorias. O tempo de internação foi dividido para análise em até três dias; de quatro a sete dias e superior a sete dias de internação.

Os dados foram analisados pelo programa computacional SAS ${ }^{23}$ (Statistical Analysis System), e comparados entre os grupos de doenças, sendo utilizado o teste quiquadrado para a comparação de proporções ou teste exato de Fisher, quando necessário, com nível de significância de $5 \%(\mathrm{P}<0,05)$.

\section{RESULTADOS}

A maioria dos pacientes estudados (45,4\%) apresentava IMC dentro da normalidade; $30,1 \%$ sobrepeso e apenas $8,1 \%$ desnutrição, com valores de $\mathrm{IMC}<18,5$. Os pacien- tes com trauma, seguidos daqueles portadores de doenças do trato digestório e abdome agudo, apresentaram IMC mais baixos, com diferença significativa entre os grupos $(P=0,0222)$ (Tabela 1$)$, e entre os que mais apresentaram perda de peso recente na internação, estavam os pacientes com abdome agudo, com diferença significativa quando comparados com os outros grupos de doenças $(P=0,0048)$ (Tabela 1). Na análise da contagem de linfócitos, foi observado que a maioria dos pacientes estudados, não apresentava depleção; no entanto quando analisado pelo grupo de doenças avaliadas, verificou-se maior percentual de depleção imunológica grave e moderada nos pacientes portadores de abdome agudo ( $\mathrm{P}<0,0001)$, e depleção leve naqueles com doenças vasculares (Tabela 1 ).

$\mathrm{Na}$ Tabela 2 foi analisado o consumo energético habitual da população estudada, entre os grupos de doenças, verificando-se maior percentual de pacientes com consumo energético inferior a 1000 kcalorias, entre aqueles com doenças vasculares, com diferença significativa entre os grupos $(P=0,0311)$.

Com relação ao tempo de internação, foi verificada diferença significativa $(\mathrm{P}<0,0001)$ no percentual entre os grupos, mostrando que pacientes com trauma foram aqueles que apresentaram maior tempo de internação (mais que sete dias), seguidos daqueles com doenças vasculares (Tabela 3).

\section{DISCUSSÃO}

O presente estudo não mostrou maior prevalência de pacientes desnutridos em enfermaria de cirurgia no período pré-operatório, apontando maior percentagem da população atendida classificada como eutrófica ou com sobrepeso. Talvez, um fator limitante deste trabalho, tenha sido o fato de não incluir-se na análise os portadores de neoplasias, o que poderia ter influenciado tais resultados. E, apesar de nos indicadores de risco nutricional estudado a maioria dos pacientes não apresentarem depleção, é importante salientar a grande percentagem de pacientes com perda de peso recente $(45,1 \%)$ encontrada. Outros dados para análise posterior, seria investigar a presença de complicações pós-operatórias naqueles pacientes com risco nutricional. Kodera, et al. ${ }^{11}$, analisando dados de 523 pacientes em estudo prospectivo randomizado, demonstraram que pancreatectomia e tempo prolongado de cirurgia foram os fatores de risco mais importantes para complicações gerais, enquanto que o IMC $\geq 25$, pancreatectomia e idade maior que 65 anos foram preditores significativos de maiores complicações cirúrgicas ${ }^{11}$.

No presente trabalho, foi observado maior tempo de internação naqueles com trauma e doenças vasculares, e maior depleção nutricional quanto aos indicadores de IMC, perda de peso recente, contagem de linfócitos e consumo energético habitual, nos pacientes portadores de trauma, abdome agudo e doenças vasculares, respectivamente. Em estudo apontando $24,2 \%$ de desnutrição, entre todos os pacientes avaliados portadores de diversas doenças, foi verificado associação entre o diagnóstico e a desnutrição, 
com prevalência significativamente maior em doenças malignas do que nas não-malignas (50.9 versus $21.0 \%$, $P<0.0001)^{20}$.

Os dados encontrados no presente trabalho mostraram altos índices de perda de peso - $45,12 \%$ da população total estudada apresentaram perda de peso recente. Este é um dado preocupante, sendo o maior percentual de perda de peso recente encontrado nos pacientes portadores de abdome agudo $(P=0,0048)$. É importante citar que a função imunológica, começa a deteriorar quando a perda de peso excede $15 \%{ }^{18}$.

Tem sido sugerido que 10 dias é o tempo suficiente para haver alteração no estado nutricional de um paciente, devendo a necessidade de intervenção ser diagnosticada tão precoce quanto possível ${ }^{17}$. Entretanto, o momento certo e as prioridades para a intervenção da EMTN continuam alvo de estudos ${ }^{22}$.

Outro achado neste trabalho, a contagem de linfócitos, mostrou depleção imunológica grave e moderada mais frequente nos pacientes com abdome agudo, com diferença significativa entre os grupos $(P<0,0001)$.

Quando verificado os diversos indicadores avaliados, entre os grupos de doenças mais frequentes estudados, foi possível observar que a maioria deles não apresentava desnutrição, apenas $8,1 \%$ eram desnutridos segundo o IMC, 54,8\% não tinham perda de peso recente e $67,4 \%$ não apresentaram depleção da contagem de linfócitos. É importante destacar que na população estudada, não estavam incluídos os pacientes portadores de neoplasias, que geralmente encontram-se desnutridos ou apresentam perda de peso ${ }^{4,14,25}$. E estavam incluídos na análise também pacientes com doenças ginecológicas, que na maioria das vezes estão com sobrepeso ou obesidade.

A desnutrição hospitalar tem sido alvo de diversos estudos nos últimos anos e, na literatura tem sido mostrado prevalência de 30 a $50 \%$ deste estado nutricional entre os pacientes internados ${ }^{3,8,13,20}$. Isso evidencia que o IMC rep- resenta um indicador muito pouco sensível à desnutrição hospitalar, pois não é sensível aos processos agudos de desnutrição, como aquele que ocorre na perda de peso não intencional entre indivíduos com reservas adiposas, como no caso de grande parte da população do presente estudo.

Se considerar-se como em risco nutricional os indicadores de $\mathrm{IMC}<18,5$, perda de peso recente, depleção grave da contagem de linfócitos, consumo energético habitual inferior a 1000 kcalorias, os dados encontrados no presente trabalho apontaram $8,1 \%, 45,1 \%, 9,4 \%$ e $14,3 \%$ dos pacientes, respectivamente, em situação de risco e que merecem maior monitoramento nutricional durante a internação. Sabe-se, de acordo com as diretrizes da ES$\mathrm{PEN}^{26}$ e outros dados recentes ${ }^{2}$ que pacientes gravemente desnutridos apresentam maiores riscos de complicações. No entanto, em estudo recente ${ }^{19}$ pacientes com desnutrição grave (albumina sérica $<3,0 \mathrm{~g} / \mathrm{dL}$ ou perda ponderal $>10 \%$ ou IMC $<18,5$ ), não mostraram significativamente maior incidência de complicações pós-operatórias.

Considerando que pacientes desnutridos podem apresentar maior morbidade e mortalidade pós-operatória, com presença de infecção de ferida cirúrgica, deiscência de anastomose, sepse e dificuldade de cicatrização quando comparado com pacientes com bom estado nutricional, estes dados confirmam a necessidade da investigação do estado nutricional no período pré-operatório, bem como a importância da aferição do peso do paciente e a necessidade de sua associação com outros indicadores nos processos de triagem e monitoramento nutricional.

\section{CONCLUSÃO}

Os pacientes portadores de abdome agudo e trauma apresentaram maior risco nutricional no período préoperatório, o que deveria demandar estratégias de atenção nutricional logo no início da internação.

Leandro-Merhi VA, Aquino JLB, Chagas JFS. Nutritional risk in the preoperative period. ABCD Arq Bras Cir Dig 2009;22(3):143-6

ABSTRACT - Background-Bad nutricional status in preoperative period - mainly associated to weight loss -, may lead to grater number of postoperative complications. Aim - To investigate the nutritional risk indicators in preoperative period, stratified by disease groups treated at the site. Methods - A total of 512 patients with the following conditions were assessed: acute abdomen, digestive tract diseases, biliary diseases, gynecological diseases, vascular diseases, hernias and traumas. The following data were analyzed: body mass index, recent weight loss, habitual energy intake, lymphocyte count and length of hospital stay. The data were compared among the disease groups. The chi-square test or Fisher's exact test when necessary was used to compare proportions. The significance level was set at $5 \%$. Results - Trauma patients followed by those with digestive tract diseases and acute abdomen had significantly lower body mass index $(P=0.0222)$, and the ones with acute abdomen presented recent weight loss at hospital admittance $(P=0.0048)$. A greater percentage of severe and moderate immune depletion was seen in patients with acute abdomen $(P<0.0001)$ and those with vascular diseases had a greater percentage of patients with energy intake below 1000 kcalories $(P=0.0311)$. Trauma patients presented longer hospital stays $(P<0.0001)$. Conclusion - Trauma and acute abdomen patients had greater nutritional risk during the preoperative period. Thus, nutritional care strategies should be oriented towards them since the hospital admittance.

HEADINGS - Nutritional status.

\section{REFERÊNCIAS}

1. Allison SP. Malnutrition, disease, and outcome. Nutrition 2000; 16:590-3.

2. Bozzetti F, Gianotti L, Braga M, Di Carloc V, Marianid L. Postoperative complications in gastrointestinal cancer patients: The joint role of the nutritional status and the nutritional support. Clin Nutr 2007; 26: 698-709.
3. Correia IMTD, Waitzberg DL. The impact of malnutrition on morbidity, mortality, length of hospital stay and costs evaluat through a multivariate model analysis. Clinical Nutrition 2003; 22(3): 235-239.

4. Dias VM, Barreto APM, Coelho SC, Ferreira FMB, Vieira GBS, Cláudio MM, Silva PDG. O grau de interferência dos sintomas gastrintestinais no estado nutricional do paciente com cancer em tratamento quimioterápico. Rev Bras Nutr Clin 2006; 21(3):211-8. 
5. Fleury. Manual de Exames 2008/2009. Laboratório Fleury. São Paulo, 2008

6. Garcia RWD, Leandro-Merhi VA, Pereira AM. Estado nutricional e sua evolução em pacientes internados em clínica médica. Revista Brasileira de Nutrição Clínica 2004; 19(2): 59-63.

7. Gil-Rendo A, Hernandez-Lizoain JL, Martinez-Regueira F, et al. Risk factors related to oerative undergoing gastrectomy for gastric câncer. Clin Transl Oncol 2006; 8:354-61.

8. Guaitoli PMR, Bottoni A, Sanchez Neto R, Sallum PM, Benedetti H, Hiroshi R, Martins-Fadiga HC, Liontakis IM, Fernandes JMR, Waitzberg DL. Avaliação do estado nutricional de pacientes adultos sob terapia nutricional internados em Unidade de Terapia Intensiva Neurológica. Rev Bras Nutr Clin 2007; 22(3): 194-6.

9. Gutiérrez Reyes JG. Serralde Zúñiga A, Guevara Cruz M. Prevalencia de desnutrición Del adulto mayor al ingreso hospitalario. Nutrición Hospitalaria 2007; 22:702-9.

10. Johansson U, Rasmussen HH, Mowe M, Staun M. Clinical nutrition in medical gastroenterology: room for improvement. Clinical Nutrition 2009; 28:129-133.

11. Kodera Y, Sasako M, Yamamoto S et al. Gastric Cancer Surgery Study Group of Japan Clinical Oncology Group. Identification of risk factors for the development of complications following extended and superextended lymphadenectomies for gastric cancer. Br J Surg 2005; 92:1103-9.

12. Kyle UG, Kossovsky MP, Karsegard VL, Pichard C. Comparison of tools for nutritional assessment and screening at hospital admission: a population study. Clinical Nutrition 2006; 25:409-417.

13. Kyle UG, Pirlich M, Lochs H, Schuetz T, Pichard C. Increased length of hospital stay in underweight and overweight patients at hospital admission: a controlled population study. Clin Nutr 2005; 24: 33-42.

14. Leandro-Merhi VA, Tristão AP, Moretto MC, Fugulin NM, Mclellan KCP, Aquino JLB. Estudo comparativo de indicadores nutricionais em pacientes com neoplasias do trato digestório. ABCD Arq Bras Cir Dig 2008; 21(3):114-9.

15. Lipschitz DA. Screening for nutritional status in the elderly. Prim Care 1994; 22(1):55-67.
16. Martins C, Moreira SM, Pierosan SR. Interações droga nutriente. $2^{\mathrm{a}}$ ed. Curitiba, Nutroclínica, 2003.

17. O'Flynn J, Peake H, Hickson M, Foster D, Frost G. The prevalence of malnutrition in hospitals can be reduced: Results from three consecutive cross-sectional studies. Clin Nutr 2005; 24: 1078-1088.

18. Osada J, Kamocki Z, Rusak M, Dabrowska M, Kedra B. The effect of surgical and nutritional treatment on activation parameters of peripheral blood $\mathrm{T}$ lymphocytes in stomach cancer patients in postoperative period. Pol Merkur Lekarski 2008; 24(141):231-6.

19. Pacelli F, Bossola M, Rosa F, Tortorelli AP, Papa V, Doglietto GB. Is malnutrition still a risk factor of postoperative complications in gastric cancer surgery? Clinical Nutrition 2008; 27:398-407.

20. Pirlich M, Schütz T, Kemps M, Luhman N, Burmester GR, Baumann G, Plauth M, Lübke HJ, Lochs H. Prevalence of malnutrition in hospitalized medical patients: impact of underlying disease. Dig Dis. 2003; 21(3):245-51.

21. Putwatana P, Reodecha P, Sirapongam Y, et al. Nutrition screening tools and the prediction of postoperative infectious and wound complications: comparison of methods in presence of risk adjustment. Nutrition 2005; 21:691-7.

22. Rasmussen HH, Kondrup J, Staun M, Ladefoged K, Lindorff K, Jorgensen LM, Jakobsen J, Kristensen H, Wengler A. A method for implementation of nutritional therapy in hospitals. Clin Nutr 2006; 25: 515-523.

23. SAS System for Windows (Statistical Analysis System), versão 9.1.3 Service Pack 3. SAS Institute Inc, 2002-2003, Cary, NC, USA.

24. Sungurtekin $\mathrm{H}$, Sungurtekin U, Balci C, et al. The influence of nutritional status on complications after major intraabdominal surgery. Journal of the American College of Nutrition 2004; 23:227-32.

25. Ulsenheimer A, Silva ACP, Fortuna FV. Perfil nutricional de pacientes com câncer segundo diferentes indicadores de avaliação. Rev Bras Nutr Clin 2007; 22(4):292-7.

26. Weimann A, Braga M, Harsanyi L, Laviano A, Ljungqvist O, Soeters P. ESPEN guidelines on enteral nutrition: surgery including organ transplantation. Clinical Nutrition 2006; 25:224-44.

27. World Health Organization. The World Health Report 1998: Life in the 21st century a vision for all. Geneva: WHO; 1998. p. 61-111.

Fonte de financiamento: não há Conflito de interesse: não há Recebido para publicação: 20/03/2009 Aceito para publicação: 28/09/2009 